\title{
Tagungsforum
}

\section{Studieneingangsphase in der Rechtswissenschaft: Jahrestagung des Zentrums für rechtswissenschaftliche Fachdidaktik Hamburg}

\author{
Lukas Musumeci"
}

Am 26./27. März 2013 fand die mittlerweile dritte Jahrestagung des Zentrums für rechtswissenschaftliche Fachdidaktik an der Universität Hamburg statt. Die Tagung widmete sich der Studieneingangsphase. Damit trägt sie zu einem ebenso traditionellen wie aktuellen hochschuldidaktischen Diskurs bei. Die Studieneingangsphase gehört seit jeher zum Themenkanon der Hochschuldidaktik und ist zur Zeit Gegenstand zahlreicher Initiativen und Projekte.

Die Tagung bestand aus fünf Themenblöcken:

- Grundlegungen

- Internationale Perspektiven

- Interdisziplinäre Perspektiven

- Die Studieneingangsphase gestalten

- Ausblick

Die Vorträge wurden am ersten Tag durch ein Weltcafé ergänzt, welches den Tagungsteilnehmern ermöglichte, sich auszutauschen und einzubringen. Weiter fanden am zweiten Tag Werkstattberichte und Projektvorstellungen statt. ${ }^{1}$

\section{A. Studieneingangsphase als Sozialisierungsphase}

Interessanterweise wurde die Tagung nicht durch eine Definition oder zumindest Umschreibung des Begriffs der Studieneingangsphase eingeleitet, wie man es gerade von Juristen erwarten könnte. Vielmehr schienen die rund 100 Tagungsteilnehmer stillschweigend zumindest über die Konturen des Begriffs übereinzustimmen. Dieser stillschweigende Konsens wurde dann auch erst am Rande der Schlussdiskussion sichtbar gemacht. Der Begriff Studieneingangsphase meint mehr als eine Orientierungseinheit oder eine Freshmen's Week. Er bezeichnet einen längeren Zeitraum zu Beginn des Studiums, der sich üblicherweise über die ersten beiden Semester erstreckt. Wo heute bereits eine curriculare Abgrenzung der Studieneingangsphase vorgenommen wurde, finden sich verschiedene Begriffe dafür wie etwa Erstjahreskurs, Assessmentjahr oder Grundstudium. Nun wäre es obsolet, eigens eine solche Phase abzugrenzen, hätte sie keine weitere eigenständige Bedeutung. Was unterscheidet also die Studieneingangsphase vom weiteren Studium?

* Der Autor war Assistent an der Universität Basel (Prof. Dr. A. Peters) und ist dort Lehrbeauftragter.

1 Das detaillierte Programm mit weiteren Unterlagen, etwa den Visualisierungen zu einzelnen Vorträgen und Werkstattberichten finden Sie auf http://www.jura.uni-hamburg.de/rechtsdidaktik/jahrestagung-2013 (1.6.2013). 
Die Studieneingangsphase wird hier als eine Sozialisierungsphase betrachtet. Sie ist eine Antwort auf die Zäsur, die der Eintritt ins Studium darstellt. Zäsuren und Übergänge bergen, wie Johannes Wildt in seinem Schlusswort heraushob, immer Risiken und Chancen in sich, insbesondere Lernchancen. Der Beginn des rechtswissenschaftlichen Studiums ist ein Übergang in zweifacher Hinsicht. Einerseits treten die Studierenden in eine neue Lebens- und Lernsituation ein. Andererseits müssen sie einen Zugang zur Rechtswissenschaft, ihrer Fachkultur und Arbeitsformen finden. Entsprechend muss die Studieneingangsphase zweifach sozialisieren. Diese frühe Sozialisierung prägt das Verständnis für das Hochschulstudium und die Rechtswissenschaften entscheidend. Somit heißt über die Studieneingangsphase sprechen über das Hochschulstudium an sich und über die Rechtswissenschaft als Ganzes zu sprechen. Dies wurde an der Tagung eindrücklich sichtbar, zumal sich viele Beiträge Eigenschaften und Problemen des rechtswissenschaftlichen Studiums annahmen, deren Bedeutung über die Studieneingangsphase hinausgeht.

\section{B. Wo liegen die Schwierigkeiten?}

Um die Studieneingangsphase zu gestalten und positiv auf die Sozialisierung einzuwirken, müssen wir die konkreten Schwierigkeiten und Herausforderungen unserer Studierenden zu Studienbeginn kennen. Lena Stadler und Roland Broemel haben diesbezüglich bei den Jurastudierenden in Hamburg nachgefragt. Ihre empirische Studie fördert zutage, dass die Schwierigkeiten in beiden Sozialisierungsebenen liegen. In der neuen Lebens- und Lernwelt bereiten das anonyme Umfeld, die Studienplanung, die Selbstorganisation und das Lernen an sich Probleme. Dieser Befund mag nicht weiter erstaunen, liegen doch in diesen Punkten gewichtige Unterschiede zur Lebens- und Lernwelt Gymnasium. ${ }^{2}$ Dem Zugang zur Rechtswissenschaft, so die Studie, stehen vor allem der mangelnde Zugang zur juristischen Methode und die Schwierigkeit, die Zusammenhänge zwischen den Rechtsgebieten zu erkennen, entgegen.

Will die juristische Ausbildung die Sozialisierung ins Studium unterstützen, so muss sie über dogmatische Inhalte hinaus Studierkompetenz und Methodenkompetenz vermitteln. Die didaktische Forschung geht davon aus, dass Kompetenzvermittlung am nachhaltigsten wirkt, wenn sie in den Unterricht der dogmatischen Inhalte eingebettet wird. Häufig ist dies an juristischen Fakultäten aber nicht der Fall. Ziel müsste es sein, diese eigenständigen Kompetenzkurse in die inhaltlichen Veranstaltungen zu integrieren. Dabei stellen die vorherrschenden institutionellen Strukturen oft ein beachtliches Hindernis dar. Es scheint in einem ersten Schritt empfehlenswert, innerhalb der existierenden Rahmenbedingungen anzusetzen.

Weiter zeigt die Studie, dass bestimmte Gruppen Studierender, etwa Studierende mit Migrationshintergrund, spezifische Probleme aufweisen. Die Sozialisierung in der

2 Siehe etwa Lagodny, Einige Überlegungen zum Studienbeginn, http://www.uni-salzburg.at/pls/portal/ docs/1/1411290.PDF (1.6.2013). Otto Lagodny arbeitet in diesem an die neuen Studierenden gerichteten Text einfach und anschaulich die Herausforderungen der neuen Lebens- und Lernwelt heraus. 
Studieneingangsphase zu unterstützen ist somit auch ein Instrument der Universitäten mit Diversität im Studierendenkörper umzugehen und diese zu fördern.

\section{Keine Angst vor dem Studium}

Wiederholt wurde an der Tagung das Problem der Angst angesprochen. Angst hemmt die Sozialisation und das Lernen. Rolf Schulmeister etwa mahnte, dass angstgesteuertes Lernen schief ginge. Dennoch sei an den Universitäten eine gewisse Angstkultur festzustellen, wie die Fachschaftsvertreterin Constanze Gütz in der Schlussdiskussion anmahnte. Angst werde geschürt von Dozierenden, von den Prüfungsämtern, von Kommilitonen und von externen Repetitoren. $\mathrm{Zu}$ beachten ist, dass die (Prüfungs-)Angst der Studierenden das Geschäftsmodell der Repetitoren ist. Auf die Angst ging auch Arnd-Christian Kulow in seiner systemischen Betrachtung des Lehr-Lernprozesses ein. Angst erzeuge das Bedürfnis nach Geborgenheit. Die Universitäten müssten die Studierenden auffangen. Dazu schlägt Kulow Beziehungsangebote zu Personen, zum Fach und zur Institution vor. Gütze betonte, dass deshalb die Zusammenarbeit zwischen den Fakultäten und den Fachschaften wichtig sei, da letztere diese Geborgenheit vermitteln und das Gemeinschaftsgefühl stärken könnten, was auch die Motivation fördere. In diese Kerbe schlägt auch die Aufforderung von Michael H. Schwartz Studierende zu integrieren, indem man sie als Kollegen behandelt.

Anzumerken sei an dieser Stelle, dass Beziehungsangebote das Potential haben, zweiseitig zu wirken. Sie ermöglichen es Dozierenden, ihre Studierenden und deren Situation, Bedürfnisse und Motivation kennen zu lernen. Dies kann dazu führen, dass sie das Bild, welches sie sich von den Studierenden gemacht haben, korrigieren oder zumindest präzisieren. Schließlich können wir nicht studierendenzentriert unterrichten, ohne zu wissen, wer unsere Studierenden sind.

\section{Grundlagen als Sozialisierung in die Rechtswissenschaft}

In mehreren Beiträgen wurde betont, wie wichtig der Aufbau reflexiver Kompetenzen für die Sozialisierung in die Rechtswissenschaft sei. Herauszuheben ist an dieser Stelle das engagierte und pointierte Plädoyer von Julian Krüper für ein grundlagenorientiertes Studium. Grundlagen ermöglichten eine Distanzierung vom geltenden Recht und, verbunden damit, von der Anwenderperspektive. Sie machten die Relativität rechtlicher Erkenntnisse und Lösungen sichtbar. Kurz, Grundlagen böten einen eigenen Blick aufs Recht. All dies ermögliche es erst das Recht kritisch zu hinterfragen. Wertbildung könne nur geschehen, wenn die Rechtsanwendung nicht zum technokratischen Akt werde.

Damit tragen die Grundlagen zur juristischen Bildung verstanden im Sinne von Nora Rzadowski bei. Ausgehend von dem klassischen Bildungsbegriff Humboldts und dem modernen Kollers sieht Rzadowski Bildungsprozesse als Transformationsprozesse. Bildung verändere wechselseitig das Verhältnis von Person und Welt. Die ju- 
ristische Ausbildung wirke somit bildend, wenn die Rechtswissenschaft den Studierenden neue Perspektiven eröffnet. ${ }^{3}$

Zudem erfüllt eine grundlagenorientierte Ausbildung die Forderung von Tilman Repgen. Dieser betonte in seiner Begrüßung, dass das Studium nicht nur gesetzliche, sondern rechtliche Lösungen vermitteln solle. Dazu müsse es aber über die Vermittlung des positiven Rechts hinausgehen und sich der Gerechtigkeit zuwenden. Repgens Aufruf kann als Forderung nach juristischer Bildung im Sinne Rzadowskis verstanden werden.

Weiter wies Krüper darauf hin, dass die Grundlagenfächer auch die kognitive Sozialisation beeinflussen. Es liessen sich zwei Kognitionstypen unterscheiden: die holistischen und die strukturellen. Holistische Kognitionstypen interessieren sich für das Ganze, für die Zusammenhänge. Sie orientieren sich an Prinzipien. Strukturelle Kognitionstypen hingegen bearbeiten Probleme isoliert. Sie orientieren sich an Regeln. Die Grundlagen, so Krüper, förderten den Sinn für die Zusammenhänge. Eine grundlagenferne Ausbildung fördere somit die strukturellen Kognitionstypen und führe zu einer kognitiven Monokultur. Der Befund von Stadler und Broemel, dass Studierende häufig Schwierigkeiten bekunden, die Zusammenhänge zwischen den Rechtsgebieten zu erkennen, verleiht der Forderung nach einem grundlagenorientierten Studium weiter Nachdruck.

Die Frage nach einer grundlagennahen oder grundlagenfernen Ausbildung ist letzten Endes die Frage danach, was für Juristen wir wollen und wie wir die Rolle des Juristen in der Gesellschaft sehen.

\section{E. Stoffreduktion als Voraussetzung}

Wie sollen aber mehr Grundlagen, mehr Methode, mehr Kompetenzen im ohnehin überfrachteten Studium ihren Platz finden? Der Weg dazu kann nur über eine planmässige Reduktion des Stoffes führen. Dies bedingt ein Nachdenken über die dogmatischen Lehrinhalte und über das Verhältnis zwischen Vorlesung, AG und Selbststudium. In Deutschland erscheint diesbezüglich das Staatsexamen als weiteres Hindernis. Sinnvollerweise können die Universitäten den Stoff nur in Zusammenarbeit mit den Prüfungsämtern reduzieren. Ansonsten wird die Abstimmung von Lernzielen, Unterricht und Prüfung, das Constructive Alignment, ${ }^{4}$ weiter reduziert. Dies würde zu einer Steigerung der Angst und zu einer Schwächung der Universitäten gegenüber den externen Repetitoren führen.

3 Auf den vielschichtigen Vortrag Rzadowskis kann hier leider nicht weiter eingegangen werden. Empfohlen sei daher der folgende Blogbeitrag, welcher die Gedanken des Vortrages in kondensierter Form wiedergibt. Rzadowski, Der Wissenschaftsrat empfiehlt: Die juristischen Fakultäten sollen Konzepte „Juristischer Bildung“" entwickeln, http://www.juwiss.de/tag/nora-rzadkowski (1.6.2013).

4 Siehe dazu Biggs, Enhancing teaching through constructive alignment, in: Higher Education 32 (1996), S. 347 . 


\section{F. Ausblick}

Was kann die Fachdidaktik für die Verbesserung der Studieneingangsphase leisten? Zunächst muss sie für das Problem sensibilisieren. Die Idee einer Studieneingangsphase, deren Hauptfunktion die Sozialisierung der Studierenden ist, harrt noch ihrer Breitenwirkung. Weiter bedarf es zusätzlicher empirischer Forschung, um die konkreten Schwierigkeiten der Studierenden zu benennen, insbesondere um Bedürfnisse spezifischer Gruppen zu erkennen. Diese Forschung mag auch die Sensibilisierung unterstützen. Die Fachdidaktik muss zudem die Möglichkeiten aufzeigen, wie die Sozialisierung innerhalb der existierenden Rahmenbedingungen verbessert werden kann. In diesem Zusammenhang seien die Werkstattberichte an der Tagung erwähnt. Diese haben eindrücklich und ermutigend aufgezeigt, wie schon heute an verschiedenen deutschen Universitäten konkret an der Verbesserung der Studieneingangsphase gearbeitet wird. Schließlich soll die Fachdidaktik, einhergehend mit der Sensibilisierung, auf eine Veränderung dieser Rahmenbedingungen hinwirken. 


\section{Studien zum Schul- und Bildungsrecht}

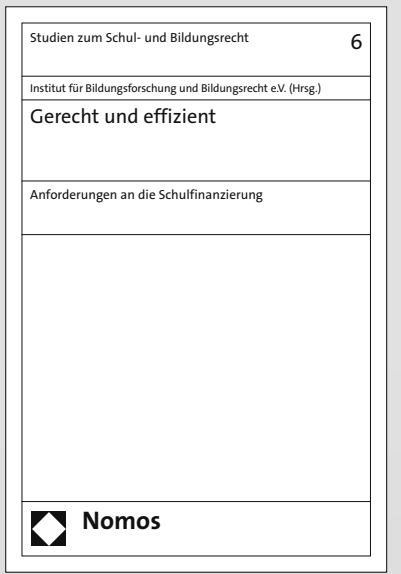

Sinkende Schülerzahlen führen zur Schließung staatlicher Schulen. „Versorgungslücken“ werden teils durch Schulen in freier Trägerschaft geschlossen. Diese Entwicklungen geben mehr

\section{Gerecht und effizient}

Anforderungen an die Schulfinanzierung Herausgegeben vom Institut für Bildungsforschung und Bildungsrecht e.V. 2013, Band 6, ca. 80 S., brosch., ca. 22,- $€$ ISBN 978-3-8487-0512-2

Erscheint ca. Oktober 2013

www.nomos-shop.de/20998

denn je Anlass, die in einigen Bundesländern erheblichen Reduzierungen der Finanzhilfe für Freie Schulen näher zu analysieren.

Weitere Bände der Reihe

\section{Zur Rechtsstellung der Lehrkräfte - heute}

1. Deutscher Schulrechtstag

Herausgegeben vom Institut für Bildungsforschung und Bildungsrecht e.V. und Deutsches Institut für Internationale Pädagogische Forschung

2013, Band 5, 95 S., brosch., 26,- $€$ ISBN 978-3-8487-0508-5

www.nomos-shop.de/20989

\section{Öffentliche Schulen in staatlicher} und freier Trägerschaft

Ausgewählte Beiträge zum Schul- und

Bildungsrecht von Johann Peter Vogel

Herausgegeben vom Institut für

Bildungsforschung und Bildungsrecht e.V.

2012, Band 4, 197 S., brosch., 54,- $€$

ISBN 978-3-8329-7960-7

wWw.nomos-shop.de/19855 


\section{Studien zum sozialen Dasein der Person}

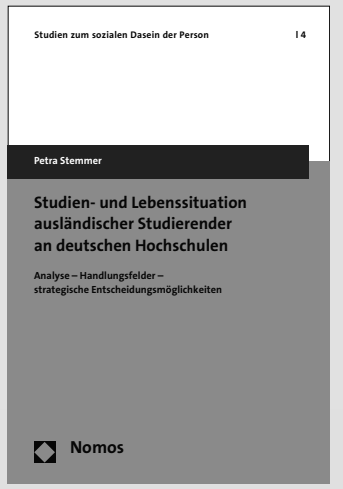

Studien- und Lebenssituation ausländischer Studierender an deutschen Hochschulen Analyse - Handlungsfelder strategische Entscheidungsmöglichkeiten

Von Petra Stemmer, MBA

2013, Band 4, ca. 300 S., brosch., 54,- $€$

ISBN 978-3-8487-0372-2

Erscheint ca. September 2013

www.nomos-shop.de/20948

Hochschulpolitik unter Druck: Die Situation ausländischer Studierender-Bildungsausländer wie Bildungsinländer-angesichts hoher Studienabbruchquoten ist kritsch. Was ist bekannt über die tatsächlichen Bedürfnisse der Studierenden?

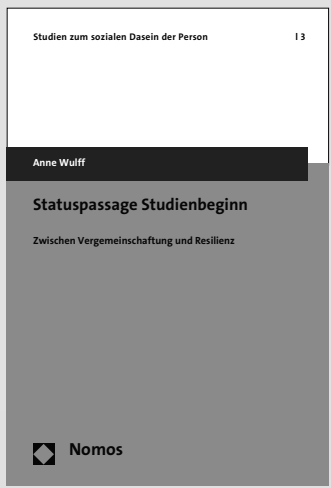

\section{Statuspassage Studienbeginn}

Zwischen Vergemeinschaftung und Resilienz Von Anne Wulff, M.A. 2013, Band 3, 92 S., brosch., 19,-€

ISBN 978-3-8487-0127-8

www.nomos-shop.de/20364

Der Studienbeginn wird aus Sicht der Transitionsforschung als Statuspassage im Lebenslauf verstanden. Das als kritisch empfundene Lebensereignis bedarf zur Bewältigung der sozio-emotionalen Anpassung. Die (Recherche-)Studie beleuchtet die Betreuungs- und Vergemeinschaftungsangebote deutscher Universitäten zum Studienbeginn.

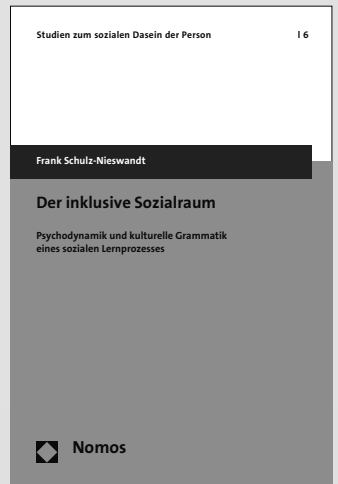

\section{Der inklusive Sozialraum}

Psychodynamik und kulturelle Grammatik eines sozialen Lernprozesses Von Prof. Dr. Frank SchulzNieswandt 2013, Band 6, 62 S., brosch., 19,-€ ISBN 978-3-8487-0777-5 www.nomos-shop.de/21522 Wie geht die Gesellschaft mit dem Andersartigen des homo patiens in der Altenpflege, der Behindertenhilfe, der Psychiatrie etc. um? Die Studie skizziert die De-Institutionalisierungsprozesse im Lichte der Rechtsphilosophie der sozialen Inklusion als schwierigen sozialen Lernprozess. 


\section{Nomos bei UTB}

\section{Forschungsprojekte entwickeln - von der Idee bis zur Publikation}

Von Prof. Dr. Waldemar Bauer,

Prof. Dr. Jörn Bleck-Neuhaus, Prof. Dr. Rainer

Dombois und Prof. Dr. Ingo S. Wehrtmann

2013, ca. 200 S., brosch., ca. 24,99€

ISBN 978-3-8252-4019-6

\section{Erscheint ca. Oktober 2013}

Für eigene Forschungsprojekt braucht es neben wissenschaftlicher Kompetenz auch Know-how im Projektmanagement u.a.. In diesem Buch vermitteln erfahrene Wissenschaftler, was es dabei zu beachten gilt und wo Fallstricke liegen.

\section{Familienrecht}

Eine sozialwissenschaftlich

orientierte Darstellung

Von Prof. Dr. Johannes Münder, RiKG Prof. Dr. Rüdiger Ernst und Prof. Dr. Wolfgang Behlert 7. Auflage 2013, ca. 350 S., brosch., ca. 24,99€ ISBN 978-3-8252-3942-8

Erscheint ca. September 2013

Das Buch wendet sich an alle, die sich systematisch mit dem Familienrecht befassen. Es stellt das Familienrecht umfassend und sozialwissenschaftlich reflektiert dar. Der Schwerpunkt liegt bei den Regelungen des BGB.

\section{Handelsrecht}

Von RA Artur Teichmann

3. Auflage 2013, 583 S., brosch., 22,99€

ISBN 978-3-8252-3763-9

Lebensnah und leicht verständlich führt Artur Teichmann in das Handelsrecht ein, sodass sowohl Wirtschaftswissenschaftler als auch Juristen einen leichten Zugang zu dieser komplexen Materie finden. Zahlreiche Beispiele, Grafiken und Lernhilfen sorgen für rasches Verstehen. Der Lernerfolg wird durch Kontrollfragen gesichert. Wo dies erforderlich ist, werden auch Bezüge zum Gesellschaftsrecht hergestellt.

\section{Basistexte: Grundrechtstheorie}

Herausgegeben von Dr. Dr. Ino Augsberg und Dr. Sebastian Unger

2012, 413 S., brosch., 19,99€

ISBN 978-3-8252-3722-6

Ohne Kenntnis des theoretischen Hintergrunds der Grundrechte ist ein angemessenes Verständnis des geltenden Verfassungsrechts nicht möglich. Der Band präsentiert die für das Verständnis der heutigen Grundrechtskonzeption entscheidenden Texte und erläutert ihre Relevanz für die daran anschließenden Diskussionen.

Bitte bestellen Sie die UTB-Nomos Titel ausschließlich bei Ihrer Buchhandlung oder unter www.utb-shop.de 


\section{Wir unterstützen Sie}

von Anfang an bei der Veröffentlichung Ihrer wissenschaftlichen Arbeiten.
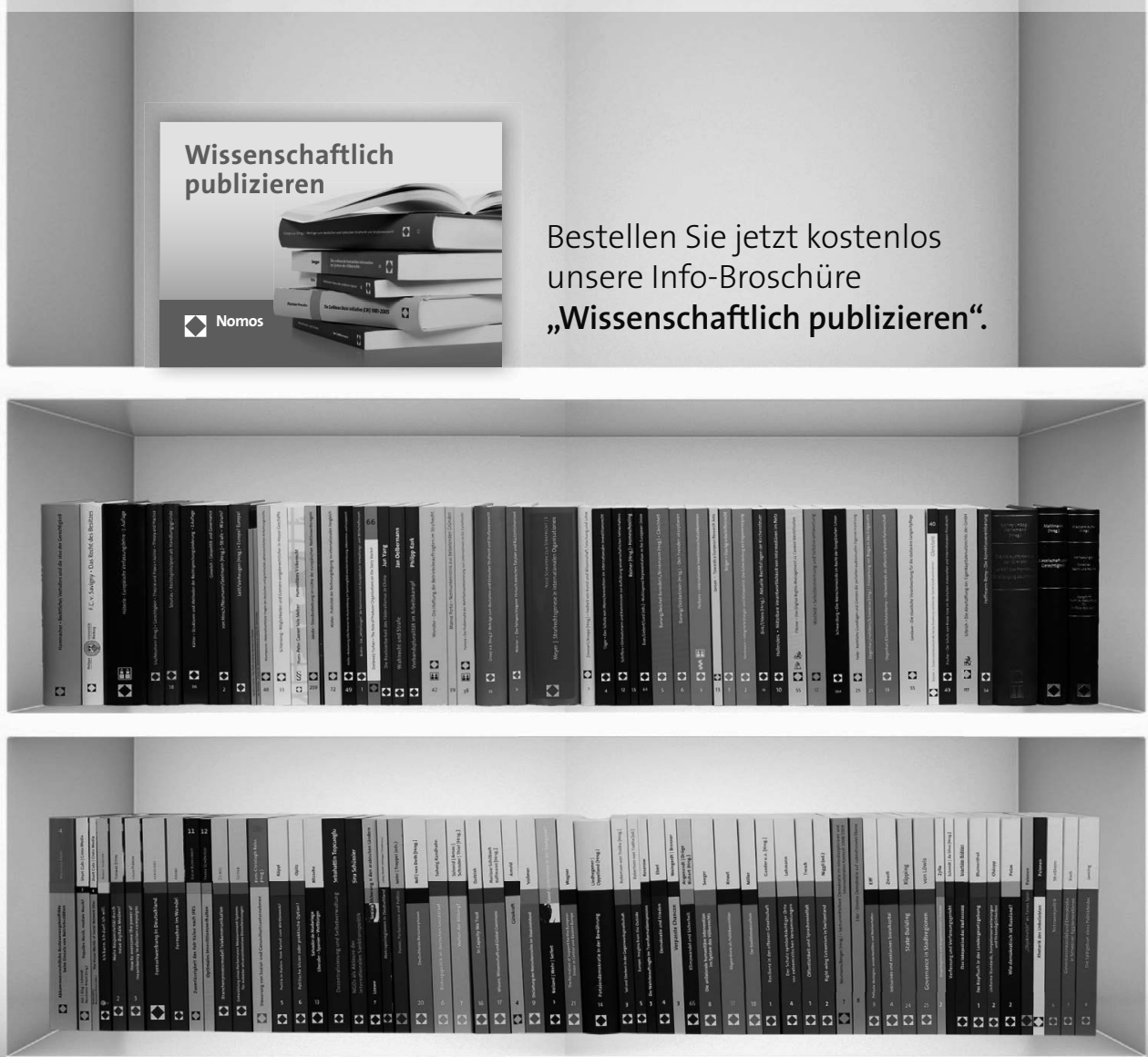

Ihr Ansprechpartner im Verlag:

Dr. Philipp Küsgens

kuesgens@nomos.de

Telefon 07221/2104-62

Nomos Verlagsgesellschaft 76520 Baden-Baden www.wissenschaftlich-publizieren.nomos.de

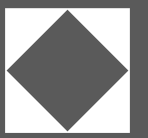

